\title{
(1) cossphas \\ Comparison of a pressure-sensing sheath and radial arterial line for intraoperative blood pressure monitoring in neurointerventional procedures
}

\author{
Michael T Froehler, Rohan Chitale, Jordan A Magarik, Matthew R Fusco
}

Cerebrovascular Program, Vanderbilt University Medical Center, Nashville, Tennessee, USA

\section{Correspondence to} Dr Michael T Froehler, Cerebrovascular Program, Vanderbilt University, Nashville, TN 37232, USA; m.froehler@ vanderbilt.edu

Received 12 January 2018 Revised 6 February 2018 Accepted 8 February 2018 Published Online First 27 February 2018
Check for updates

To cite: Froehler MT, Chitale R, Magarik JA, et al. J Neurolntervent Surg 2018; 10:784-787.

\section{ABSTRACT \\ Purpose The efficiency of neuroendovascular} procedures may partly depend on the time devoted to placement of a radial arterial line (RAL) for intraoperative blood pressure monitoring. An alternative approach is to use a pressure-sensing sheath (PSS) that serves to provide invasive blood pressure monitoring without requiring a separate procedure for placement. We compared the use of a RAL versus PSS and assessed procedure time, anesthetist and patient satisfaction, and cost.

Methods We performed a single-center, prospective, blockwise, comparative trial of procedure start time using traditional RAL placement versus the EndoPhys PSS for invasive blood pressure monitoring. Endpoints included time from room arrival to groin puncture, patient and anesthetist satisfaction ratings, and costs associated with RAL placement.

Results Twenty patients were enrolled in the PSS+RAL arm and 20 in the PSS-alone arm. Mean time from arrival in the room until groin puncture was $61.9 \pm 14.0 \mathrm{~min}$ in the RAL group and $51.2 \pm 10.8 \mathrm{~min}$ in the PSS-alone group ( $P=0.01$; difference $=10.7 \mathrm{~min}$ ). Patients in the PSS-alone group reported less pain than those in the RAL group. Furthermore, anesthetists reported accurate blood pressure in the PSS group. The average cost estimate of RAL placement was US\$774.70, with a range of US\$743 to US\$1171.

Conclusions Placement of a RAL at the start of the neuroendovascular procedures resulted in increased delays to procedure start time and more patientreported pain compared with the PSS, which may offer a more efficient means of blood pressure monitoring for neurointerventional procedures.

Clinical trial registration NCT03239847.

\section{INTRODUCTION}

Continuous blood pressure monitoring during neuroendovascular procedures offers multiple advantages, including real-time hemodynamic monitoring and rapid treatment of blood pressure variations. Rapid treatment of elevated blood pressure is critical during endovascular treatment of intracranial aneurysms and arteriovenous malformations. Traditionally, continuous invasive blood pressure monitoring has been achieved with a percutaneous catheter, typically a radial arterial line (RAL). While a RAL can provide real-time blood pressure measurement, it does have several drawbacks. For example, the radial artery may have blood pressure that is lower than the true systemic blood pressure due to atherosclerosis and stenosis of the subclavian $\operatorname{artery}^{1-3}$ and thus may prompt unnecessary augmentation of the blood pressure. Furthermore, RAL placement may cause significant discomfort for the patient. They are typically left in place for the postoperative period, even though continuous pressure measurement is no longer needed. RAL placement also poses added intraprocedural danger related to infection and vessel injury. ${ }^{4}$ Finally, placement of a RAL can be time consuming, ${ }^{5}$ resulting in delayed procedure-start times and longer total operative times, which increases perioperative risk of surgery. ${ }^{6-9}$

A potential alternative to the RAL is the pressure-sensing sheath (PSS; EndoPhys, Dallas, TX, USA) that can be used for continuous blood pressure monitoring during transfemoral endovascular procedures. The PSS is a simple $6 \mathrm{Fr}, 10 \mathrm{~cm}$ sheath that incorporates a solid-state pressure transducer and is inserted just like a typical arterial sheath (figure 1). The PSS has been shown to provide reliable blood pressure measurement that is similar to that of a RAL. ${ }^{10}$ It is possible that the use of a PSS could potentially save time and improve operating room (angiography suite) efficiency compared with placement of a RAL, which ultimately could result in improved patient safety. Furthermore, patients may prefer this approach as it avoids the wrist discomfort associated with RAL placement.

We hypothesized that the use of a PSS would result in faster procedure-start times compared with preprocedure placement of a RAL. We conducted a prospective study to compare times of the two approaches and additionally explored patient and anesthetist satisfaction, and costs associated with the RAL.

\section{METHODS}

We conducted a single-center, prospective, blockwise comparison of blood pressure monitoring with the PSS and an RAL (PSS+RAL group) versus use of the PSS alone (PSS-alone group). The primary endpoint was the length of time from entry into the operating room until start of the procedure, as defined by groin puncture. All procedures at our institution are performed in hybrid operating rooms with biplane angiography. Times were recorded by a study coordinator, who was physically present in the operating room throughout the procedure. 
A
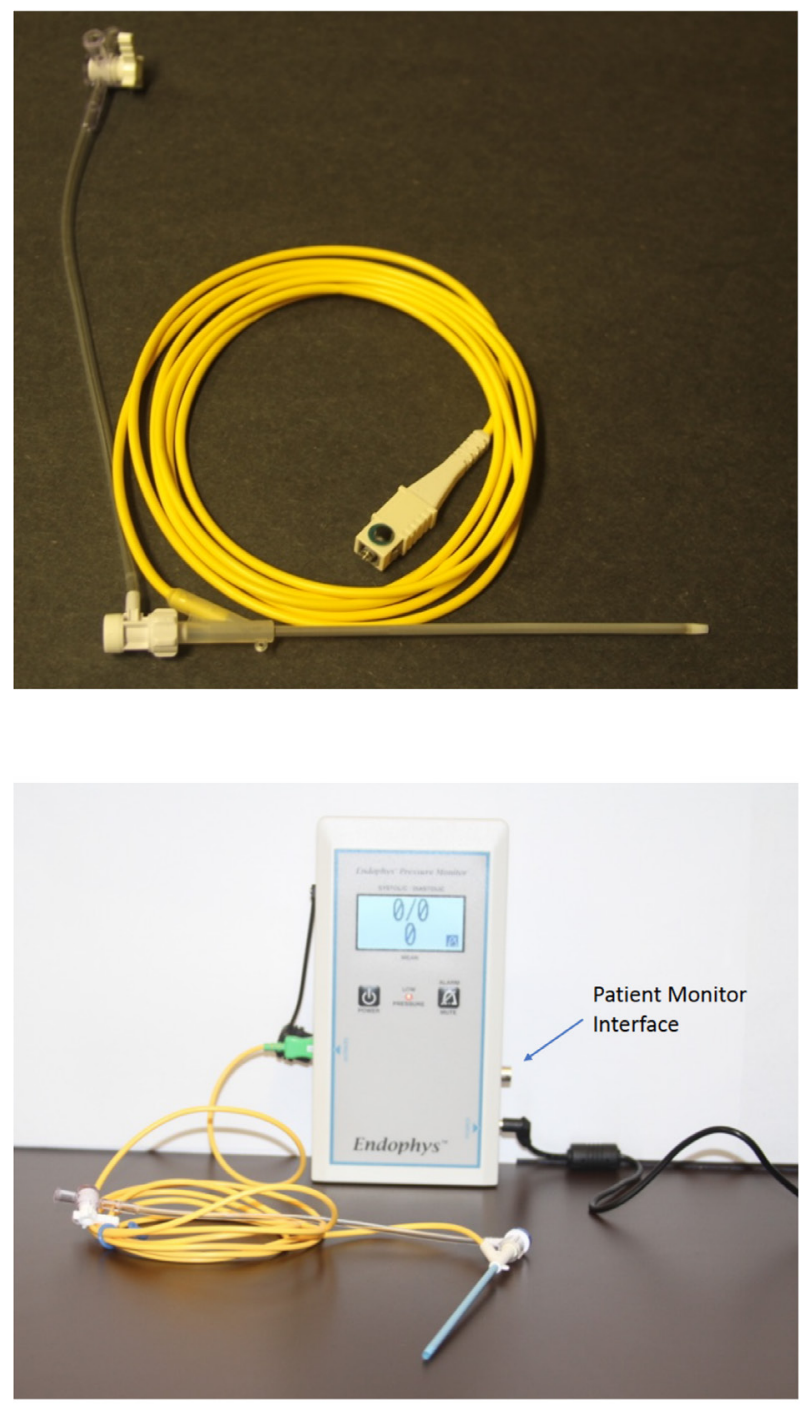

Figure 1 The EndoPhys pressure sensing sheath. A 6-Fr device is shown in panel $A$. The yellow wire attaches to the pressure monitor which is shown in Panel B, along with an 8-Fr device. The pressure monitor can interface with the typical patient monitors used in the hospital.

Cases were included if general anesthesia and continuous blood pressure monitoring were necessary for the procedure, and if access to both the radial and femoral arteries was adequate. Exclusion criteria included: contraindication for RAL placement, hemodynamic instability, or if an ongoing need for invasive blood pressure monitoring postoperatively was anticipated (such as with aneurysmal subarachnoid hemorrhage). Informed consent was obtained from all participants prior to enrollment.

The PSS is an FDA-cleared groin sheath that is similar to other arterial access sheaths, with the exception of a solid-state pressure-transduction system. ${ }^{10}$ This allows continuous, invasive blood pressure measurement without the need for a gap between the sheath and the guide catheter, that is, a 6-Fr catheter can be used in a 6-Fr sheath. All PSS devices used in the trial were 6-Fr and $10 \mathrm{~cm}$ in length and all were provided by EndoPhys. An 8-Fr sheath is available (figure 1B) but was not used in this study.

Patients were asked about their current level of pain in the wrist, maximum level of pain in the wrist, and if it was bothersome, on postoperative day 1 and at outpatient follow-up 2-4 weeks after discharge. The neuroanesthesia providers were asked to rate their satisfaction with the blood pressure reading from the sheath and if they felt that it matched the RAL (in the PSS + RAL group) or cuff (in the PSS-alone group). If there was a discrepancy, they were asked why they thought there was a difference. Anesthetists were also asked if any difficulty was encountered in RAL placement.

The cost of RAL placement was calculated based on the individual cost of all components, including equipment, professional fees, and use of ultrasound. The RAL is made by Arrow International (Teleflex, Wayne, PA, USA) and is charged at US\$235.79 for the first device and US\$67.79 for each subsequent device used in the same procedure. Additional necessary equipment included a transducer (US\$7.98), tubing (US\$12.06), drapes (US\$2.23), gloves (US\$1.47), saline bag (US\$6.41), and a chlorhexidine swab (US\$5.86). The cost for the non-pressure sensing femoral access sheath (Pinnacle, a division of Terumo Interventional Systems; Somerset, NJ, USA) was US\$33.50. The professional fee was a standard US\$168 for each RAL placement procedure, regardless of difficulty or time.

The dollar value of any additional time associated with RAL placement in the operating room was calculated as an opportunity cost, based on the assumption that additional availability of the room has value. This necessitates an estimate of the value of operating room time per minute, though these estimates vary greatly in the literature. ${ }^{11-13}$ Based on estimates of otolaryngology (US\$38/min), urology (US\$22/min), ${ }^{12}$ and cosmetic surgery (UD\$62/min), ${ }^{13}$ we chose a conservative estimate of US $\$ 2 / \mathrm{min}$ of operating room time.

\section{RESULTS}

Twenty patients were enrolled in the PSS + RAL arm and 20 in the PSS arm. Baseline characteristics did not differ between the two groups (table 1). In particular, there was no observable difference in the degree of femoral artery atherosclerosis, and the procedure type mix was similar between the two groups.

Placement of the PSS sheath in all 40 patients was successful. In general, the sheath behaves similarly to other arterial access

\begin{tabular}{|c|c|c|c|}
\hline Characteristic & RAL+PSS & PSS only & $P$ value \\
\hline Number of participants & 20 & 20 & \\
\hline Median age & 59 & 56 & 0.68 \\
\hline$\%$ female & 70 & 55 & 0.327 \\
\hline \multicolumn{4}{|l|}{ Femoral atherosclerosis } \\
\hline None & 5 & 6 & 0.88 \\
\hline Mild & 11 & 12 & \\
\hline Moderate & 1 & 0 & \\
\hline Severe & 3 & 2 & \\
\hline \multicolumn{4}{|l|}{ Procedure type } \\
\hline Aneurysm embolization & 9 & 5 & 0.45 \\
\hline AVM embolization & 3 & 6 & \\
\hline dAVF embolization & 3 & 3 & \\
\hline Tumor embolization & 2 & 2 & \\
\hline Other & 3 & 4 & \\
\hline
\end{tabular}

Other cases included three venous stenting procedures for idiopathic intracranial hypertension, two intracranial angioplasty procedures, one spinal angiogram, and one pipeline reconstruction of arterial dissection.

AVM, arteriovenous malformation; dAVF, dural arteriovenous fistula; PSS, pressuresensing sheath; RAL, radial arterial line. 
Median Door-to-Puncture time

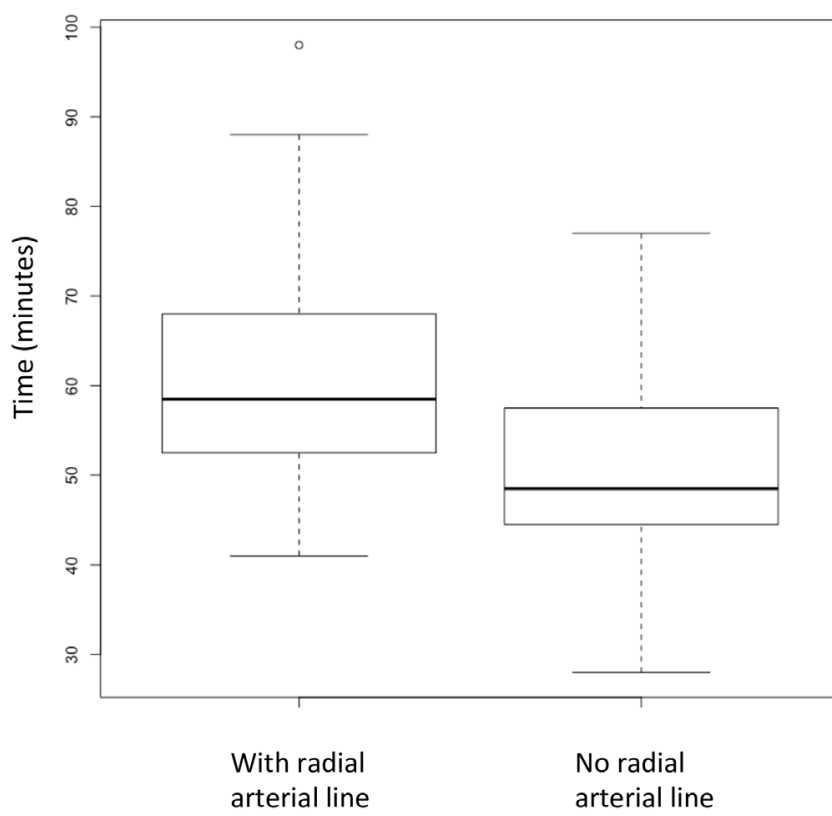

Figure 2 Boxplots of median door-to-puncture times for patients with radial arterial line (RAL) placement and use of the pressure-sensing sheath (PSS) (left) versus use of the PSS alone (right). Median time is indicated by the bold line, and first and third quartiles are indicated by the box. Whiskers indicate minima and maxima, with a single outlier in the RAL group indicated by an open circle. The median door-to-puncture time for patients with RAL was 58.5 and without RAL was 48.5.

sheaths. However, the PSS sheath does seem to benefit from a small skin incision at the puncture site prior to sheath insertion.

Case-start efficiency, as measured by the mean time from entry to the operating room until groin puncture, was $61.9 \mathrm{~min}$ $( \pm$ SD of 14.0$)$ in the RAL group and $51.2 \mathrm{~min}$ ( \pm SD of 10.8 ) in the PSS-alone group $(\mathrm{P}=0.01)$. The difference between the means is $10.7 \mathrm{~min}$, with a $95 \% \mathrm{CI}$ of 2.7 to $18.7 \mathrm{~min}$ saved by avoidance of RAL placement. Comparison of median times and

\section{Maximum patient reported pain, on a scale of 0-10}

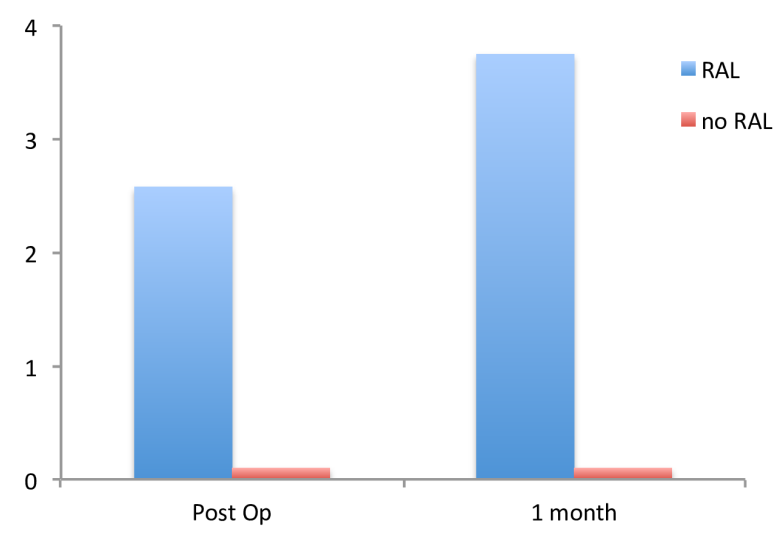

Figure 3 Average maximum pain in the wrist for patients with radial arterial line (RAL) placement $(n=19)$ versus patients with pressuresensing sheath (PSS) alone $(n=18)$. Patients without RAL placement universally denied any pain at the wrist. their distributions are seen in figure 2, with a median of 58.5 in the RAL group and 48.5 in the PSS-alone group.

In general, the anesthesiology team was satisfied with the performance of the PSS. On a scale of 1 to 10, with 10 representing complete satisfaction, the median score for performance of the PSS was 8. Likewise, anesthetists felt that the PSS generally produced similar blood pressure measurements to the RAL or cuff, where discrepancies were noted in seven out of 40 cases (four in PSS + RAL and three in PSS alone). In discrepant cases, anesthetists generally felt that the difference was attributable to regional pressure differences between the arm and leg, and not due to malfunction of the device. Anesthetists also noted difficulty in placing the RAL in five of 20 cases (eight required the use of more than one RAL catheter).

Patients reported pain associated with RAL placement. Compared with the patients who underwent RAL placement, patients that had the PSS alone reported less pain, both immediately after the procedure and at 1 month (figure 3 ). Mean pain scale rating (0-10) for the maximum amount of wrist pain experienced postoperatively was 2.6 and at 1 month was 3.75 for RAL patients $(n=19)$, whereas it was 0 postoperatively and 0 at 1 month for patients that received the PSS alone $(n=18)$. Six of the 19 patients surveyed after RAL placement reported that the pain was bothersome.

The average cost for RAL placement was calculated based on device, equipment, and professional fees. The mean cost per patient was US\$507.20, which accounts for six patients that required the use of a second RAL catheter and two patients that required the use of three RAL catheters. Based on a difference of $10.7 \mathrm{~min}$ in case-start efficiency, the opportunity cost of RAL placement was conservatively estimated to be US\$267.50. Using a range of OR cost estimates from the literature, ${ }^{12} 13$ the true value of this operating room time could range from US $\$ 235.40$ to US\$663.40. Thus, the total mean cost for RAL placement in the operating room prior to procedure start is approximately US\$774.70, with a range of US\$743 to US\$1171 based on reported OR costs. ${ }^{11-13}$

\section{DISCUSSION}

This study has shown that the use of a PSS for intraoperative blood pressure monitoring was faster than RAL placement by an average of over $10 \mathrm{~min}$. The output of the PSS was generally reliable and resulted in less patient discomfort compared with placement of a RAL.

Increasing efficiency in the operating room is a common goal. ${ }^{14-16}$ In particular, increased throughput can optimize the number of procedures performed daily, improve financial performance of the room, improve physician efficiency, and improve patient satisfaction and outcomes by reducing the length of time patients spend under general anesthesia. Our study shows that the use of a PSS instead of a RAL may result in an average time saving of over $10 \mathrm{~min}$. In addition, we found that the time to place the RAL was variable, with an IQR of $13.75 \mathrm{~min}$. In fact, several patients with RAL placement had delays to procedure start of over $80 \mathrm{~min}$, whereas none of the PSS-alone group had such long delays.

Certain types of procedures are particularly time-critical. In particular, efficiency is critical in the endovascular treatment of acute ischemic stroke. ${ }^{17}{ }^{18}$ In this study, we only included patients undergoing elective procedures. However, the time savings demonstrated here could be applied to emergent procedures such as thrombectomy for stroke. In fact, given the need for swift treatment, many proceduralists forgo the placement of a RAL before thrombectomy and instead simply rely on blood pressure 
measurement via a cuff. However, given the critical importance of blood pressure and hemodynamic management in patients with acute ischemic stroke, real-time invasive blood pressure monitoring using a PSS would be helpful. Fortunately, an 8-Fr PSS has recently become available, however, its impact on emergent thrombectomy procedures has not yet been evaluated.

If RAL placement is part of the normal routine for acute stroke treatment, a time saving of over $10 \mathrm{~min}$ could be expected to result in improved functional outcomes. ${ }^{17} 1920$ But more importantly, the variability of the time required for RAL placement suggests that some stroke procedures could be delayed by over $80 \mathrm{~min}$. This would certainly be expected to impact the rate of good functional outcome, potentially reducing the rate of good functional outcome by $7 \%$ to $30 \% .^{1721}$

The impact on patient pain and satisfaction should not be overlooked. Many studies have shown that pain is strongly linked to patient dissatisfaction. ${ }^{22-24}$ In this context, one should first examine whether continuous, invasive blood pressure monitoring is necessary. If so, as it often is with complex intracranial cerebrovascular procedures, the use of a PSS may still provide the necessary physiological monitoring while avoiding patient discomfort. In this study, we found that $6 / 19$ patients reported bothersome pain. Furthermore, the mean reported score for maximum pain actually increased from postoperative day 1 to outpatient follow-up at 1 month. This suggests that patients do not forget pain associated with their procedure, and thus their pain is likely to affect long-term satisfaction.

Anesthetist satisfaction is also important. Given the critical nature of hemodynamic factors during cerebrovascular procedures, an accurate and reliable blood pressure measurement is mandatory. A previous study has already shown the similarity between RAL and PSS blood pressure measurement. ${ }^{10}$ The current study showed that, in general, anesthetists were satisfied with either the RAL or PSS. Discrepancies were noted in $17.5 \%$ of procedures, and these differences were felt to be due to regional pressure differences attributable to atherosclerotic stenotic disease. Perhaps more important than absolute value of blood pressure measurement is the detection of change, which was consistently reliable with both the RAL and PSS throughout this study. This may partly explain the high anesthetist satisfaction scores associated with use of the PSS.

Our financial analysis showed that the cost of RAL placement is not insignificant. Particularly in light of the time disadvantage associated with RAL placement, the use of the PSS may be justified in many situations. However, the precise estimate of operating room opportunity cost is highly variable and depends on many factors that may be unique to the institution. Furthermore, the cost of the equipment itself will vary greatly from institution to institution. With these limitations and our small sample size, a true cost-effectiveness analysis is far beyond the scope of this article. Nonetheless, the results here do provide a framework for individual institutions to perform their own cost analyses for approaches to intraoperative blood pressure monitoring during neuroendovascular procedures.

This study has limitations. While we have shown that additional time was necessary for RAL placement prior to procedure start, there may be idiosyncratic reasons for this such as placement of RAL in the OR rather than in the holding room. It was also an unblinded study, which could have had some effect on outcome measurement. The RAL cost analysis is also particularly limited, given that there is likely to be significant cost variability between institutions. There is also wide variation in estimates of operating room opportunity cost, and thus such calculations must be done at the institutional level.
Contributors MTF designed the study, collected and analyzed data, and drafted the paper. He is the guarantor. RVC collected data and critically revised the paper. JAM collected data and critically revised the paper. MRF collected and analyzed data, and critically revised the paper.

Funding This work was supported by a research grant from EndoPhys Holdings, LLC.

Competing interests MTF serves as a consultant for Balt USA, Medtronic, Stryker, and Viz.ai and has received research funding from Medtronic, Stryker, Microvention, EndoPhys, and Penumbra. RVC has received research funding from Microvention and Medtronic.

Patient consent Not required.

Ethics approval Institutional Review Board.

Provenance and peer review Not commissioned; externally peer reviewed.

Open access This is an open access article distributed in accordance with the Creative Commons Attribution Non Commercial (CC BY-NC 4.0) license, which permits others to distribute, remix, adapt, build upon this work non-commercially, and license their derivative works on different terms, provided the original work is properly cited, appropriate credit is given, any changes made indicated, and the use is non-commercial. See: http://creativecommons.org/licenses/by-nc/4.0/.

(C) Article author(s) (or their employer(s) unless otherwise stated in the text of the article) 2018. All rights reserved. No commercial use is permitted unless otherwise expressly granted.

\section{REFERENCES}

1 Semina LI, lunes AM. Asymmetry of arterial blood pressure in the brachial artery in patients with cerebrovascular pathology. Zh Nevropatol Psikhiatr Im S S Korsakova 1992;92:27-31.

2 Langwieser N, Prechtl L, Meidert AS, et al. Radial artery applanation tonometry for continuous noninvasive arterial blood pressure monitoring in the cardiac intensive care unit. Clin Res Cardiol 2015;104:518-24.

$3 \mathrm{Kim} \mathrm{SH}$, Lilot M, Sidhu KS, et al. Accuracy and precision of continuous noninvasive arterial pressure monitoring compared with invasive arterial pressure: a systematic review and meta-analysis. Anesthesiology 2014;120:1080-97.

4 Scheer B, Perel A, Pfeiffer UJ. Clinical review: complications and risk factors of peripheral arterial catheters used for haemodynamic monitoring in anaesthesia and intensive care medicine. Crit Care 2002;6:199-204

5 Shiloh AL, Savel RH, Paulin LM, et al. Ultrasound-guided catheterization of the radial artery: a systematic review and meta-analysis of randomized controlled trials. Chest 2011;139:524-9.

6 Qiu CS, Jordan SW, Dorfman RG, et al. Surgical duration impacts venous thromboembolism risk in microsurgical breast reconstruction. J Reconstr Microsurg 2018:34:047-58

7 Phan K, Kim JS, Capua JD, et al. Impact of operation time on 30-day complications after adult spinal deformity surgery. Global Spine J 2017;7:664-71.

8 Bekelis K, Labropoulos N, Coy S. Risk of venous thromboembolism and operative duration in patients undergoing neurosurgical procedures. Neurosurgery 2017;80:787-92.

9 Kim JY, Khavanin N, Rambachan A, et al. Surgical duration and risk of venous thromboembolism. JAMA Surg 2015;150:110-7.

10 Purdy PD, South C, Klucznik RP, et al. Use of a pressure sensing sheath: comparison with standard means of blood pressure monitoring in catheterization procedures. J Neurointerv Surg 2017;9:766-71.

11 Macario A. What does one minute of operating room time cost? J Clin Anesth 2010;22:233-6.

12 Chatterjee A, Payette MJ, Demas CP, et al. Opportunity cost: a systematic application to surgery. Surgery 2009;146:18-22.

13 Shippert RD. A study of time-dependent operating room fees and how to save $\$ 100$ 000 by using time-saving products. Am J Cosmetic Surg 2005;22:25-34.

14 Pedron S, Winter V, Oppel EM, et al. Operating room efficiency before and after entrance in a benchmarking program for surgical process data. J Med Syst 2017:41:151.

15 Tagge EP, Thirumoorthi AS, Lenart J, et al. Improving operating room efficiency in academic children's hospital using Lean Six Sigma methodology. J Pediatr Surg 2017:52:1040-4

16 Healey T, El-Othmani MM, Healey J, et al. Improving operating room efficiency, part 1: general managerial and preoperative strategies. JBJS Rev 2015;3: 10

17 Goyal M, Jadhav AP, Bonafe A, et al. Analysis of workflow and time to treatment and the effects on outcome in endovascular treatment of acute ischemic stroke: results from the swift prime randomized controlled trial. Radiology 2016:279:888-97.

18 Menon BK, Sajobi TT, Zhang Y, et al. Analysis of workflow and time to treatment on thrombectomy outcome in the endovascular treatment for small core and proximal occlusion ischemic stroke (escape) randomized, controlled trial. Circulation 2016;133:2279-86. 
19 Khatri P, Yeatts SD, Mazighi M, et al. Time to angiographic reperfusion and clinical outcome after acute ischaemic stroke: an analysis of data from the Interventional Management of Stroke (IMS III) phase 3 trial. Lancet Neurol 2014;13:567-74.

20 Prabhakaran S, Ruff I, Bernstein RA. Acute stroke intervention: a systematic review. JAMA 2015;313:1451-62.

21 Froehler MT, Saver JL, Zaidat 00, et al. Interhospital transfer before thrombectomy is associated with delayed treatment and worse outcome in the STRATIS registry (Systematic Evaluation of Patients Treated With Neurothrombectomy Devices for Acute Ischemic Stroke). Circulation 2017;136:2311-21.
22 Gunaratne R, Pratt DN, Banda J, et al. Patient dissatisfaction following total knee arthroplasty: a systematic review of the literature. J Arthroplasty 2017;32:3854-60

23 Howells N, Murray J, Wylde V, et al. Persistent pain after knee replacement: do factors associated with pain vary with degree of patient dissatisfaction? Osteoarthritis Cartilage 2016;24:2061-8.

24 Bertrand LA, Voelzke BB, Elliott SP, et al. Measuring and predicting patient dissatisfaction after anterior urethroplasty using patient reported outcomes measures. J Urol 2016;196:453-61. 